\title{
Transgenic Killer Commensal Bacteria as Mucosal Protectants
}

\author{
Luciano Polonelli \\ Dipartimento di Patologia e Medicina di Laboratorio, Sezione di Microbiologia, \\ Università degli Studi di Parma, Viale A. Gramsci 14, 43100 Parma, Italy
}

KEY WORDS: killer recombinant antibodies, transgenic commensal bacteria, mucosal immunotherapy

DOMAINS: applied microbiology; infection; drug delivery; molecular biology

As first line of defense against the majority of infections and primary site for their transmission, mucosal surfaces of the oral cavity and genitourinary, gastrointestinal, and respiratory tracts represent the most suitable sites to deliver protective agents for the prevention of infectious diseases. Mucosal protection is important not only for life threatening diseases but also for opportunistic infections which currently represent a serious burden in terms of morbidity, mortality, and cost of cures. Candida albicans is among the most prevalent causes of mucosal infections not only in immunocompromised patients, such as HIV-infected subjects who are frequently affected by oral and esophageal candidiasis, but also in otherwise healthy individuals, as in the case of acute vaginitis. Unfortunately, current strategies for mucosal protection against candidiasis are severely limited by the lack of effective vaccines and the relative paucity and toxicity of commercially available antifungal drugs.

An additional option has been reported in a recent issue of Nature Biotechnology [1]. By exploiting the idiotypic network, the investigators first generated a recombinant antiidiotypic antibody in the single chain format representing the internal image of a yeast killer toxin characterized by the wide spectrum of antimicrobial activity (KTscFv) [2]. $\mathrm{KTscFv}$, through its ability to mimic the yeast killer toxin activity, exerted a therapeutic effect against $C$. albicans rat vaginal infection. $\mathrm{KTscFv}$, moreover, mimics the functional activity of protective microbicidal antibodies naturally produced during candidal infection in humans [3]. The peculiarity of the new experimental approach is the combination of such exclusive antibiotic KTscFv with a unique delivery system constituted by a naturally transformable commensal bacterium (Streptococcus gordonii) which was engineered for secreting or displaying at its surface KTscFv. This delivery system, based on a live recombinant microorganism, was chosen since $S$. gordonii is able to express heterologous proteins efficiently during mucosal colonization and deliver candidate vaccines to a mammalian host for oral and vaginal immunization [4].

Thus, engineered killer commensal bacteria were used for the first time therapeutically for colonizing rat vaginal mucous membranes to eradicate an experimental candidal infection. In vivo data showed that recombinant $S$. gordonii, particularly in its KTscFv secreting form, was as effective as a full course of fluconazole in rapidly abating the fungal burden.

One of the main problems connected with the evaluation of the clinical efficacy of antibodies on mucosal surfaces is their high cost and limited production capacity. The short half-life of these molecules demands continuous administration and raises problems of bioavailability. The steady mucosal delivery of microbicidal antibodies permanently expressed by colonizing commensal bacteria is judged of primary importance for a prospective therapeutic intervention. In the study recombinant commensal bacteria persisted to colonize the rat vagina for at least 15 days. This consolidated animal model includes, among its advantages, the requirement of persistent bacterial colonization, and in its estrogen dependence, characteristic invasive hyphal Candida growth in loco and vaginal $\mathrm{T}$ lymphocyte assets, 
has a strong degree of similarity to human infection [5]. As such, it has been adopted to test the preclinical actrivity of antimycotic and immunotherapeutic agents.

Even though vaginal candidiasis represents an ideal experimental model of mucosal infections, the rationale of the new technology should be applicable to other infections, caused by pathogenic microorganisms proven to be susceptible to the yeast killer toxin, such as Cryptococcus neoformans, Pneumocystis carinii, and multidrug-resistant strains of Mycobacterium tuberculosis, on all mucosal surfaces [6].

The use of other appropriate recombinant mucosal bacteria such as Lactobacillus spp. to deliver therapeutic antibodies or peptides may represent an exciting and potentially general strategy for the safe treatment and prevention of different mucosal infections.

In the perspective of clinical trials, however, it remains to be ascertained whether long-term colonization of the human mucosal surfaces with recombinant commensal bacteria is completely safe. Moreover, the means for large scale production and the regulatory pathway for new transgenic commensals will need to be defined.

\section{REFERENCES}

1. Beninati, C., Oggioni, M.R., Boccanera, M., Spinosa, M.R., Maggi, T., Conti, S., Magliani, W., De Bernardis, F., Teti, G., Cassone, A., Pozzi, G., and Polonelli, L. (2000) Therapy of mucosal candidiasis by expression of an anti-idiotype in human commensal bacteria. Nat. Biotechnol. 18, 1060-1064.

2. Magliani, W., Conti, S., De Bernardis, F., Gerloni, M., Bertolotti, D., Mozzoni, P., Cassone, A., and Polonelli, L. (1997) Therapeutic potential of antiidiotypic single chain antibodies with yeast killer toxin activity. Nat. Biotechnol. 15, 155-158.

3. Polonelli, L., De Bernardis, F., Conti, S., Boccanera, M., Magliani, W., Gerloni, M., Cantelli, C., and Cassone, A. (1996) Human natural yeast killer toxin-like candidacidal antibodies. $J$. Immunol. 156, 1880-1885.

4. Medaglini, D., Rush, C.M., Sestini, P., and Pozzi, G. (1997) Commensal bacteria as vectors for mucosal vaccines against sexually transmitted diseases: vaginal colonization with recombinant streptococci induces local and systemic antibodies in mice. Vaccine $\mathbf{1 5}$, 1330-1337.

5. De Bernardis, F., Lorenzini, R., and Cassone, A. (1999) Rat model of Candida vaginal infection. In Handbook of Animal Models of Infection. Zak, O. and Sande, M.A., Eds. Academic Press, New York, pp. 735-740.

6. Magliani, W., Conti, S., Gerloni, M., Bertolotti, D., and Polonelli L. (1997) Yeast killer systems. Clin. Microbiol. Rev. 10, 369-400.

This article should be referenced as follows:

Polonelli, L. (2001) Trangenic killer commensal bacteria as mucosal protectants. TheScientificWorld 1, 196-197.

\begin{tabular}{llr}
\hline Received: & February & 27,2001 \\
Accepted: & March & 26,2001 \\
Published: & May & 1,2001
\end{tabular}



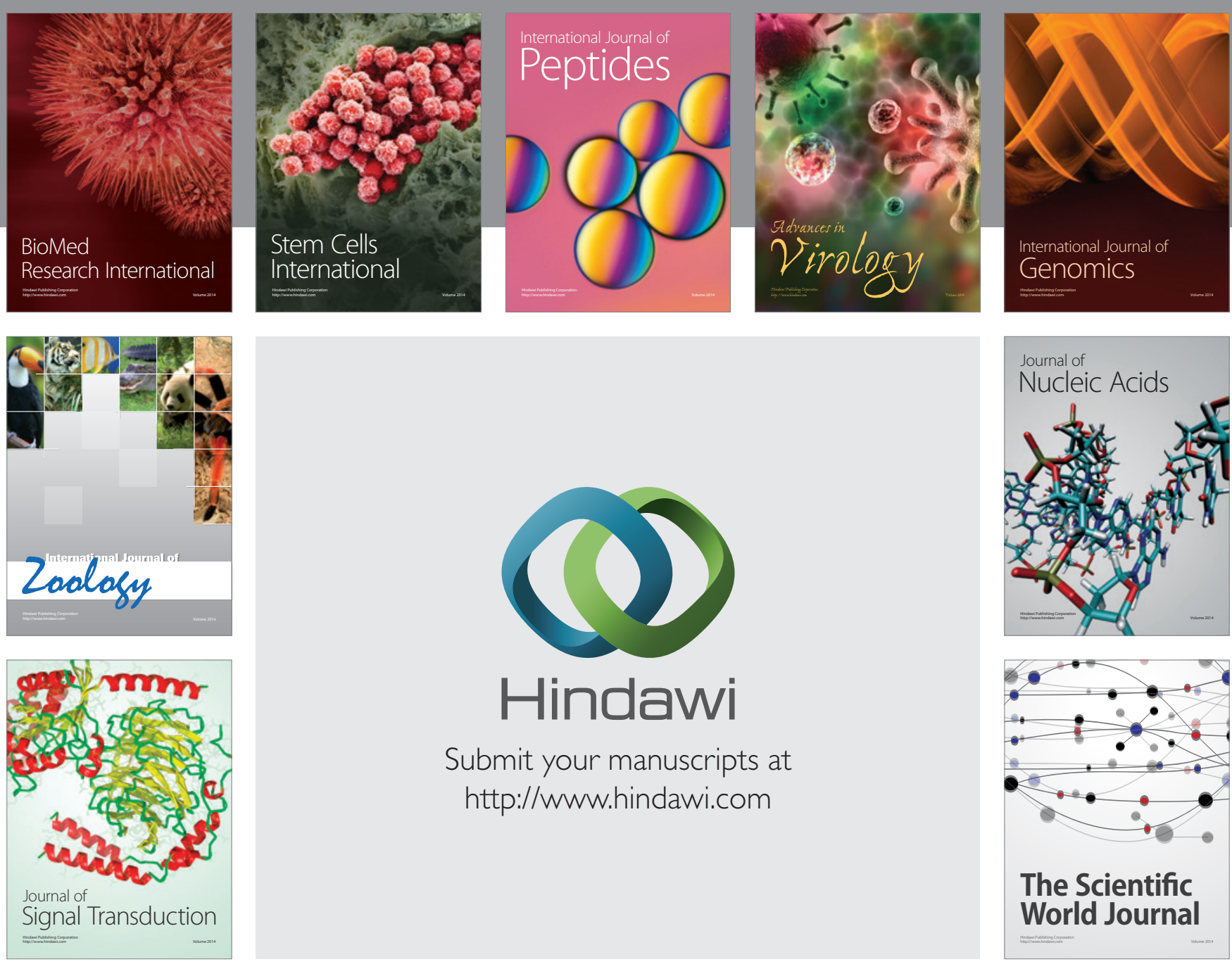

Submit your manuscripts at

http://www.hindawi.com
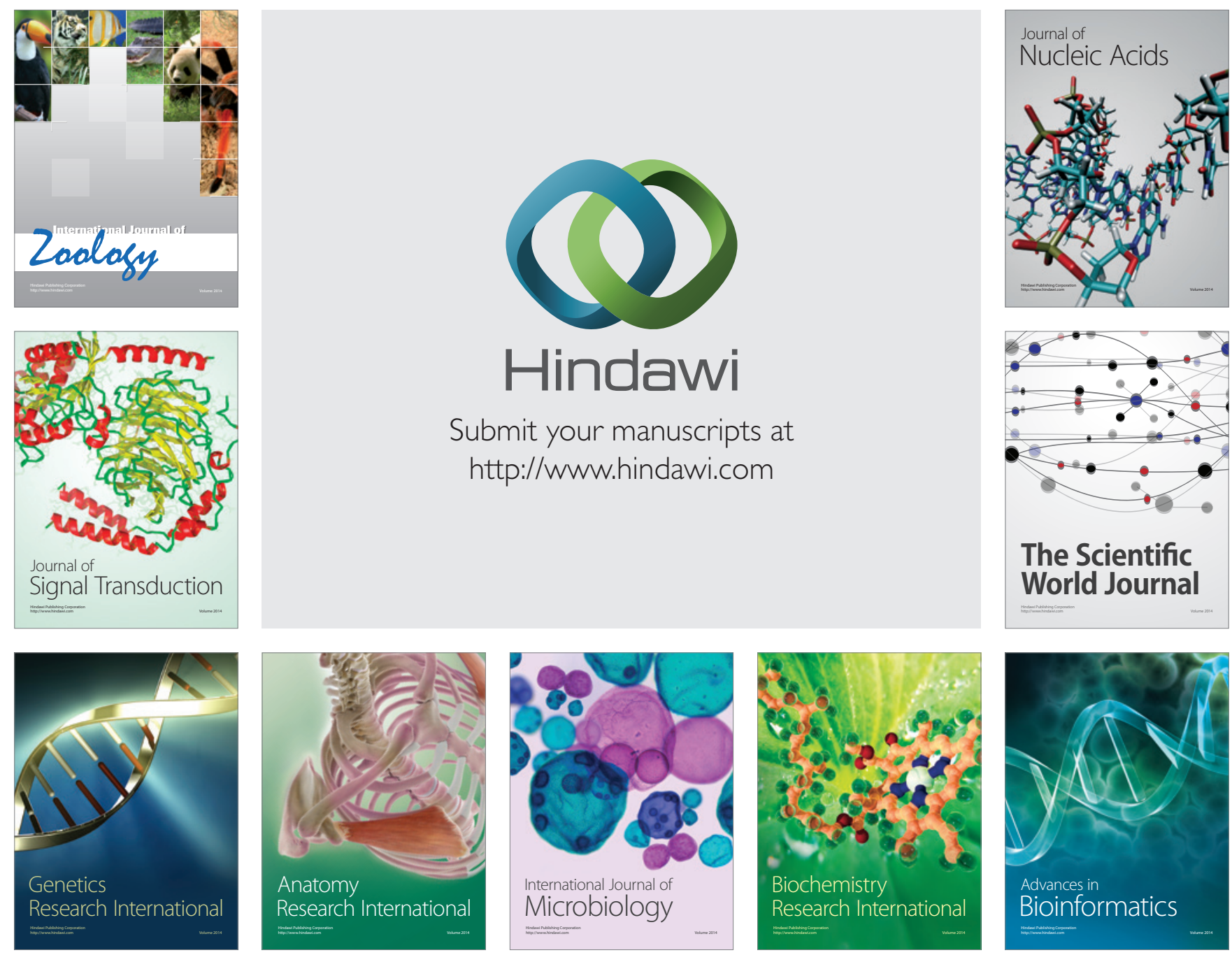

The Scientific World Journal
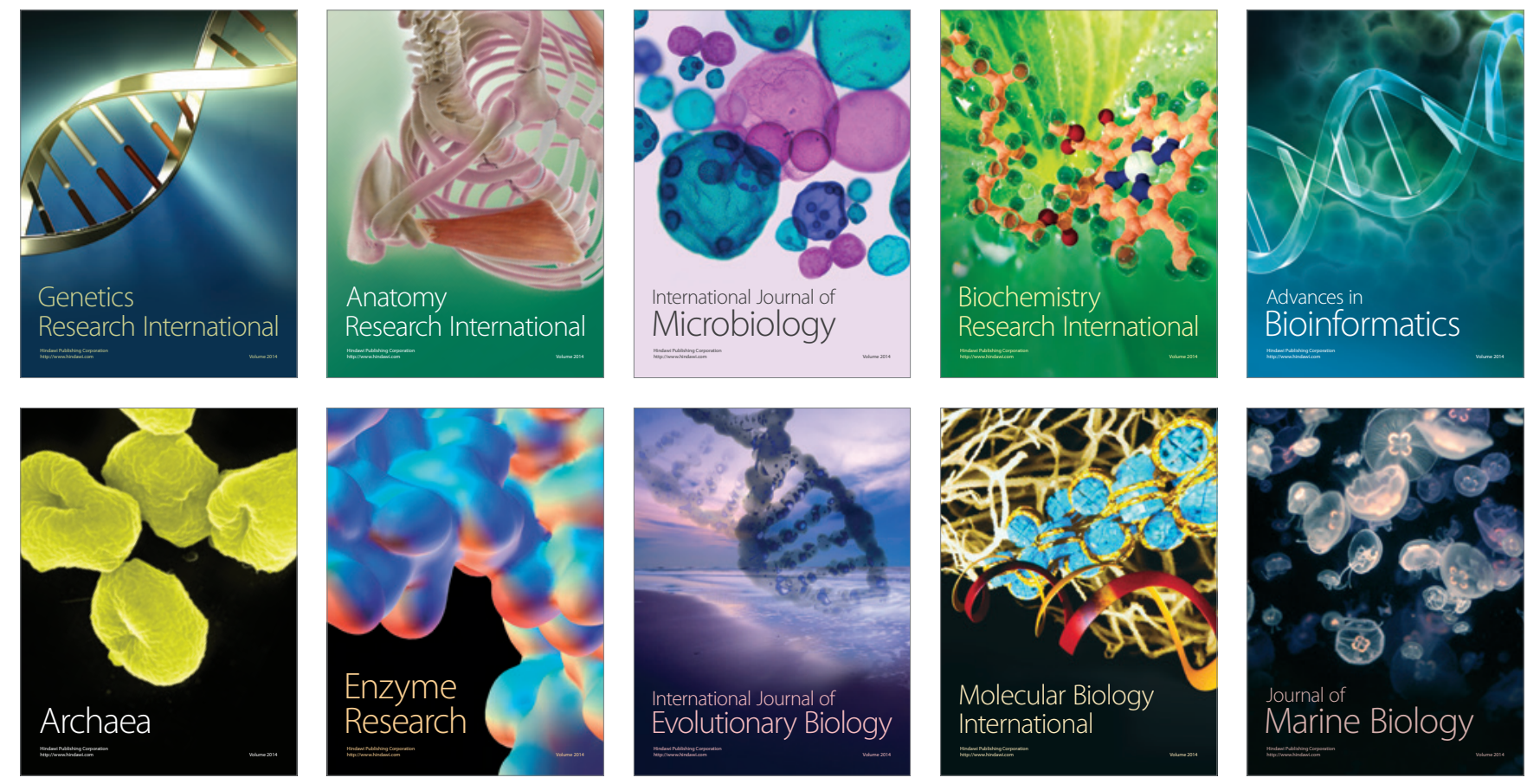